\title{
Radio-sensitising effect of T-DM1 should not be discarded for the efficacy of radiosurgery in the management of brain metastases in patients with HER2-positive metastatic breast cancer
}

\author{
Kadri Altundag ${ }^{1}$ (D)
}

Received: 21 December 2016 / Accepted: 19 March 2017 / Published online: 23 March 2017

(c) Springer Science+Business Media New York 2017

To the Editor,

I want to congratulate Geraud et al. [1] for their study. They evaluated the efficacy and safety of concurrent use of radiation therapy (RT) and T-DM1 for the treatment of brain metastases (BM) in patients with HER2-positive metastatic breast cancer (BC). They reported that the combination of T-DM1 and radiosurgery were feasible but can increase the incidence of radiation necrosis. Although authors stated that radiotherapy may increase the efficacy of T-DM1, they did not mention about the radio-sensitizing capacity of T-DM1. The tumour resistance to radiotherapy is one of the obstacles to improve cancer patients' outcomes. Certain drugs were shown to be effective to sensitize cells to RT. However, the clinical utility of potent radiosensitizing drugs is limited by their side effects in practice. There is a recent study reported potent anti-tubulin drugs conjugated to anti-ErbB antibodies; one of them, namely as T-DM1 selectively radiosensitize tumours based on surface receptor expression [2]. The authors showed that concurrent use of T-DM1 with RT prolongs tumour control in tumours overexpressing HER2. In conclusion, T-DM1 provides potent and tumour selective radio-sensitization. Having known that increased incidence of radiation necrosis when used with RT, radiosentizing capacity of T-DM1 should be further investigated in larger clinical trials.

Kadri Altundag

altundag66@yahoo.com

1 MKA Breast Cancer Clinic, Tepe Prime, 06800 Cankaya, Ankara, Turkey
Compliance with ethical standards

Conflict of interest The author indicated no potential conflicts of interest.

\section{References}

1. Geraud A, Xu HP, Beuzeboc P, Kirova YM (2016) Preliminary experience of the concurrent use of radiosurgery and T-DM1 for brain metastases in HER2-positive metastatic breast cancer. J Neurooncol 131:1-4

2. Adams SR, Yang HC, Savariar EN, Aguilera J, Crisp JL, Jones KA et al (2016) Anti-tubulin drugs conjugated to anti-ErbB antibodies selectively radiosensitize. Nat Commun 7:13019 